\title{
Tensor Formulation of Spin-1 and Spin-2 Fields
}

\author{
A. J. Macfarlane and W. Tait \\ Department of Applied Mathematics and Theoretical Physics, Cambridge, England
}

Received August 10, 1971

\begin{abstract}
Spin projection operators which constitute a resolution of the identity in the space of second rank tensor wave functions are constructed. These projectors are then used to establish Lagrangian quantum field theories for free massive particles with spin-1 (two equivalent formulations) and spin-2.
\end{abstract}

\section{Introduction}

Recently, Aurilia and Umezawa $[1,2]$ have introduced an elegant systematic and flexible method for constructing quantized field theories for free particles with higher spins. For such particles the method yields a single wave equation, which implies all the usual subsidiary conditions, including symmetry or antisymmetry properties, so that the wave function in question has exactly the number of components appropriate to its spin value. Further, in virtue of these subsidiary conditions, the original equation then reduced to the Klein-Gordan equation for integral spins and to the Dirac equation for half-integral spins. The method of Aurilia and Umezawa represents a decisive advance in the theory of higher spin particles initiated by Dirac [3], Fierz [4], and Pauli [5], developed further by Rarita and Schwinger [6] and many others, and, in fact, excellently reviewed by these authors themselves [2].

The quantization of the various wave equations in the A.U. approach, i.e. the derivation of commutation relations and Feynman propagators is achieved by introducing the operator $[7,8,9] d(p)$, known as the Klein-Gordan divisor, which has the property

$$
d(p) \Lambda(p)=\Lambda(p) d(p)=-\left(p^{2}-m^{2}\right),
$$

where the kernel $\Lambda(p)$ is given by the wave equation

$$
\Lambda(p) \psi=0 .
$$

The operator $d(p)$ is closely related to the spin projection operators $\Theta(s)$ introduced by Fronsdal [10], which project the spin $s$ components out of an arbitrary wave function. In fact $d(p)$ is equal to the projection operator when $p^{2}-m^{2}=0$. 
When the mass-shell condition is not satisfied, then the spin projection operator contains singular terms, viz. powers of $p^{-2}$, so that the wave equations introduced by Fronsdal [10]

$$
(\Theta(s) \gamma \cdot p-m) \psi=0
$$

for half-integer spin, and

$$
\left(\Theta(s) p^{2}-m^{2}\right) \phi=0,
$$

for integer spin, contain singularities which are not at all desirable, especially when interactions are introduced. Previously the removal of such singularities has been effected by the introduction of auxiliary fields, a concept suggested by Fierz and Pauli, and later developed by Fronsdal [10] and Chang [11]. In the A.U. theory, an alternative to the method of auxiliary fields is proposed which has been successful in treating vector, two- and threespinor, and vector-spinor fields.

In this paper the method is applied to a second rank tensor field $\psi^{\mu v}(x)$. In section two, all the projection operators for the different spin components contained in this tensor are derived. The anti-symmetric part is examined in section three, and two (equivalent) formulations of the theory of spin-1 in terms of anti symmetric second rank tensors are worked out. One of these formulations closely related to the Proca description of spin-1,involves some new results, and the other is a slight generalization of a recent formulation due to Takahashi and Palmer [12]. Some insight into the relationship of these two approaches is afforded by relating them to the Bargmann-Wigner equations [13]. In section four, the description of a massive spin-2 particle in terms of a second rank tensor $\psi^{\mu \nu}$ is considered. A wave equation, second order in the first derivatives of the wave function is obtained which implies all the usual subsidiary conditions for spin-2, including the symmetry and tracelessness of $\psi^{\mu v}$, and, in virtue of these, reduced to the Klein-Gordan equation. The results obtained agree with those of Bhargava and Watanabe [14]. These authors obtained their results by a different, somewhat tentative, method and with the aid of a correct but unproven assumption which we did not have to make. We believe, moreover, that our analysis is significant as an illustration of the method of Aurilia and Umezawa: indeed it is probably the simplest non-trivial example of their method, for the spin-1 examples of section three lack some of the typical complications of the general case. This is worthwhile, since, at present no theory of spins $5 / 2, \ldots$ or $3, \ldots$ free from subsidiary fields exists, and if such theories are to be found it seems most likely that the approach of Aurilia and Umezawa should yield them.

Finally it is noted that the results of Bhargava and Watanabe, which we have here confirmed in all details, contradict the work of Rivers [15], 
whose analysis implies that it is impossible to have a consistent quantized theory of spin-2 fields unless it is assumed a priori that $\psi^{\mu \nu}$ is symmetric.

\section{Projection Operators}

In this section we construct projection operators which project out of an arbitrary second-rank tensor $\psi^{\mu v}$, parts which transform irreducibly under the group of spatial rotations. It is well known that the symmetric part of $\psi^{\mu v}$ involves spin-2 and spin- 1 each once and spin- 0 twice, while the anti-symmetric part involves spin-1 twice. We seek a resolution of the identity

$$
\begin{gathered}
I=S+A, \\
S=D(2)+D(1)+D(0)+T(0), \\
A=A(1)+A^{\prime}(1),
\end{gathered}
$$

where

in an obvious notation in which the bracketed integers denote spin values. Eq. (2.1) written out in full is

$$
g_{\sigma}^{\mu} g_{\varrho}^{v}=\frac{1}{2}\left(g_{\sigma}^{\mu} g_{\varrho}^{v}+g_{\varrho}^{\mu} g_{\sigma}^{v}\right)+\frac{1}{2}\left(g_{\sigma}^{\mu} g_{\varrho}^{v}-g_{\varrho}^{\mu} g_{\sigma}^{v}\right) ;
$$

and we proceed as follows to derive explicit formulas for the projectors occurring on the right of (2.2) and (2.3).

The two elements of the centre of the enveloping algebra of the Poincaré algebra are

and

$$
p^{2}=p_{\mu} p^{\mu}
$$

where

$$
W=-w_{\mu} w^{\mu},
$$

$$
w_{\mu}=\frac{1}{2} \varepsilon_{\mu \nu \sigma \varrho} S^{\nu \sigma} p^{\varrho} .
$$

In (2.5), $\boldsymbol{S}^{\nu \sigma}$ denotes the spin operator, and we can write

$$
W=\frac{1}{2} p^{2} \boldsymbol{S}_{\mu \nu} \boldsymbol{S}^{\mu \nu}-\boldsymbol{S}_{\mu \sigma} \boldsymbol{S}^{\mu \varrho} p^{\sigma} p_{\varrho} .
$$

For massive particle-like representations, the eigenvalues of $p^{2}$ and $W$ are $m^{2}>0$ and $s(s+1) m^{2}$. Now an arbitrary tensor $\psi^{\mu v}$ carries a reducible representation involving several spins as already noted. The corresponding spin operator is

where

$$
\left(\boldsymbol{S}^{\alpha \beta}\right)_{\sigma \varrho}^{\mu \nu}=\left(\Sigma^{\alpha \beta}\right)_{\sigma}^{\mu} g_{\varrho}^{\nu}+g_{\sigma}^{\mu}\left(\Sigma^{\alpha \beta}\right)_{\varrho}^{\nu},
$$

is the spin operator for a four-vector

The first step in the construction of the required projectors is to construct projectors onto the parts of $\psi^{\mu v}$ which carry spins $2,1,0$. Using 
the standard expression

we obtain

$$
P(s)=\prod_{s^{\prime} \neq s} \frac{W-s^{\prime}\left(s^{\prime}+1\right) p^{2}}{s(s+1) p^{2}-s^{\prime}\left(s^{\prime}+1\right) p^{2}},
$$

$$
\begin{aligned}
& P(2)=\frac{W^{2}}{24 p^{4}}-\frac{W}{12 p^{2}}, \\
& P(1)=-\frac{W^{2}}{8 p^{4}}+\frac{3 W}{4 p^{2}}, \\
& P(0)=\frac{W^{2}}{12 p^{4}}-\frac{2 W}{3 p^{2}}+1 .
\end{aligned}
$$

Use of (2.6), (2.7) and (2.8) leads hence to the explicit expressions.

$$
\begin{aligned}
P(2) \equiv & D(2), \\
D(2)_{\sigma \varrho}^{\mu \nu}= & -\frac{1}{3} g^{\mu v} g_{\sigma \varrho}+\frac{1}{2}\left(g_{\sigma}^{\mu} g_{\varrho}^{v}+g_{\varrho}^{\mu} g_{\sigma}^{v}\right) \\
& +\frac{1}{3 p^{2}}\left(g^{\mu v} p_{\sigma} p_{\varrho}+p^{\mu} p^{v} g_{\sigma \varrho}\right)+\frac{2}{3 p^{4}} p^{\mu} p^{v} p_{\varrho} p_{\varrho} \\
& -\frac{1}{2 p^{2}}\left(g_{\varrho}^{v} p^{\mu} p_{\sigma}+g_{\sigma}^{\mu} p^{v} p_{\varrho}+g_{\varrho}^{\mu} p^{v} p_{\sigma}+g_{\sigma}^{v} p^{\mu} p_{\varrho}\right), \\
P(1) \equiv & A+D(1), \\
D(1)_{\sigma \varrho}^{\mu \nu}= & \frac{1}{2 p^{2}}\left(g_{\varrho}^{v} p^{\mu} p_{\sigma}+g_{\sigma}^{\mu} p^{v} p_{\varrho}+g_{\varrho}^{\mu} p^{v} p_{\sigma}+g_{\sigma}^{v} p^{\mu} p_{\varrho}\right) \\
& -\frac{2}{p^{4}} p^{\mu} p^{v} p_{\sigma} p_{\varrho}, \\
P(0)_{\sigma \varrho}^{\mu v}= & \frac{1}{3} g^{\mu v} g_{\sigma \varrho}-\frac{1}{3 p^{2}}\left(g^{\mu v} p_{\sigma} p_{\varrho}+p^{\mu} p^{v} g_{\sigma \varrho}\right) \\
& -\frac{4}{3 p^{4}} p^{\mu} p^{v} p_{\sigma} p_{\varrho} .
\end{aligned}
$$

In these equations, we have made the obvious identifications of $D(2)$ and $D(1)$ with $P(2)$ and the symmetric part of $P(1)$.

To complete the resolution, we follow the systematic approach of Aurilia and Umezawa [2]. These authors instruct us to consider all operators of the form

$$
D\left(s_{1}\right)_{\alpha}^{\mu} D\left(s_{2}\right)_{\beta}^{v} P(S)_{\sigma e}^{\alpha \beta},
$$

for $S=0,1,2, s_{1}=0,1$ and $s_{2}=0,1$. Herein, we have

$$
\begin{aligned}
& D(0)_{\alpha}^{\mu}=p^{\mu} p_{\alpha} / p^{2}, \\
& D(1)_{\alpha}^{\mu}=g_{\alpha}^{\mu}-p^{\mu} p_{\alpha} / p^{2},
\end{aligned}
$$


which are the projectors onto spin- 0 and spin- 1 for a four-vector. To explain the procedure, let us note that $\psi^{\mu \nu}$ transforms under Lorentz transformations like a product $\phi^{\mu} \chi^{v}$ of four vectors. The operator (2.13) yields the spin-S part of $\psi^{\mu v}$ which transforms like the spin- $S$ part of the direct product of the spin $s_{1}$ part of $\phi^{\mu}$ and the spin $s_{2}$ part of $\chi^{\nu}$. Since $S=2$ can only arise when $s_{1}=s_{2}=1$, the results

$$
\begin{aligned}
D(1)_{\alpha}^{\mu} D(1)_{\beta}^{v} P(2)_{\sigma \varrho}^{\alpha \beta} & =P(2)_{\sigma \varrho}^{\mu \nu}, \\
D\left(s_{1}\right)_{\alpha}^{\mu} D\left(s_{2}\right)_{\beta}^{v} P(2)_{\sigma \varrho}^{\alpha \beta} & =0, \quad \text { unless } s_{1}=s_{2}=1,
\end{aligned}
$$

confirm what we have seen already. Similarly, since $S=1$ belongs to the anti symmetric part of the direct product when $s_{1}=s_{2}=1$, we may construct the projector, $A(1)$ say, by taking

$$
\begin{aligned}
A(1)_{\sigma \varrho}^{\mu \nu}= & D(1)_{\alpha}^{\mu} D(1)_{\beta}^{v} P(1)_{\sigma \varrho}^{\alpha \beta} \\
= & \frac{1}{2}\left(g_{\sigma}^{\mu} g_{\varrho}^{v}-g_{\varrho}^{\mu} g_{\sigma}^{v}\right) \\
& -\frac{1}{2 p^{2}}\left(g_{\varrho}^{v} p^{\mu} p_{\sigma}+g_{\sigma}^{\mu} p^{v} p_{\varrho}-g_{\varrho}^{\mu} p^{v} p_{\sigma}-g_{\sigma}^{v} p^{\mu} p_{\varrho}\right) .
\end{aligned}
$$

Likewise it is natural to identify $D(1)$ and $A^{\prime}(1)$ with

$$
\frac{1}{2}\left[D(1)_{\alpha}^{\mu} D(0)_{\beta}^{v} \pm D(0)_{\alpha}^{\mu} D(1)_{\beta}^{\nu}\right] P(1)_{\sigma \varrho}^{\alpha \beta}
$$

and we check that $D(1)_{\sigma \varrho}^{\mu \nu}$ as given by (2.11) emerges, as consistency demands, and that

$$
\begin{aligned}
A^{\prime}(1)_{\sigma \varrho}^{\mu \nu} & =A_{\sigma \varrho}^{\mu \nu}-A(1)_{\sigma \varrho}^{\mu \nu} \\
& =\frac{1}{2 p^{2}}\left(g_{\varrho}^{v} p^{\mu} p_{\sigma}+g_{\sigma}^{\mu} p^{v} p_{\varrho}-g_{\varrho}^{\mu} p^{v} p_{\sigma}-g_{\sigma}^{v} p^{\mu} p_{\varrho}\right),
\end{aligned}
$$

follows. Further, $D(0)_{\alpha}^{\mu} D(0)_{\beta}^{v} P(1)_{\sigma \varrho}^{\alpha \beta}$ vanishes. Finally, turning to $S=0$, we find

$$
\begin{aligned}
D(1)_{\alpha}^{\mu} D(1)_{\beta}^{v} P(0)_{\sigma \varrho}^{\alpha \beta}= & \frac{1}{3} g^{\mu v} g_{\sigma \varrho}-\frac{1}{3 p^{2}}\left(g^{\mu v} p_{\sigma} p_{\varrho}+p^{\mu} p^{v} g_{\sigma \varrho}\right) \\
& +\frac{1}{3 p^{4}} p^{\mu} p^{v} p_{\sigma} p_{\varrho}, \\
D(0)_{\alpha}^{\mu} D(0)_{\beta}^{v} P(0)_{\sigma \varrho}^{\alpha \beta}= & p^{\mu} p^{v} p_{\sigma} p_{\varrho} / p^{4} .
\end{aligned}
$$

Since $D(1)_{\alpha}^{\mu} D(0)_{\beta}^{v} P(0)_{\sigma \varrho}^{\alpha \beta}$ and $D(0)_{\alpha}^{\mu} D(1)_{\beta}^{v} P(0)_{\sigma \varrho}^{\alpha \beta}$ vanish, it is seen that the Aurilia-Umezawa procedure [2] yields the required projectors and no more. However it is possible to resolve $P(0)$ in another way, by the trace 
operator

and

$$
\begin{gathered}
T(0)_{\sigma \varrho}^{\mu \nu}=\frac{1}{4} g^{\mu v} g_{\sigma \varrho}, \\
D(0)_{\sigma \varrho}^{\mu v}=\frac{1}{12} g^{\mu v} g_{\sigma \varrho}-\frac{1}{3 p^{2}}\left(g^{\mu v} p_{\sigma} p_{\varrho}+p^{\mu} p^{v} g_{\sigma \varrho}\right) \\
+\frac{4}{3 p^{4}} p^{\mu} p^{v} p_{\sigma} p_{\varrho} .
\end{gathered}
$$

In Sect. 4, where the kernel of the spin-2 Lagrangian is constructed it will become obvious why the latter spin- 0 projectors are used in preference to the former; in order to have an obvious proof that $\psi^{\mu v}$ is traceless. The resolution (2.1) to (2.3) is now fully specified, with

$$
D(2), D(1), D(0), T(0), A(1) \text { and } A^{\prime}(1)
$$

given respectively by Eqs. (2.10), (2.11), (2.17), (2.16), (2.14) and (2.15).

\section{The Two Descriptions of Spin-1 by Anti-Symmetric Tensors}

It is well-known that the Proca equations for spin-1 can be written in terms of an antisymmetric second rank tensor. Recently Takahashi and Palmer [12] have shown that there is an alternative formulation of spin-1 theory in terms of such a second rank tensor. This formulation is simpler in appearance but equivalent to the previous one. One way to obtain the primitive versions of these two theories is to set out from the Bargmann-Wigner equations for a symmetric second-rank spinor $\phi$. Expanding $[16] \phi$ in the form

$$
\phi=\operatorname{im} \gamma_{\lambda} C A^{\lambda}+\frac{1}{2} \sigma_{\lambda v} C F^{\lambda v},
$$

where $C$ is the charge conjugation matrix, $A^{\lambda}$ a four vector and $F^{\lambda v}$ an anti-symmetric second rank tensor, we can show that the equations

$$
\begin{aligned}
p_{v} F^{\lambda v} & =m^{2} A^{\lambda}, \\
F^{\mu v} & =p^{v} A^{\mu}-p^{\mu} A^{v}
\end{aligned}
$$

follow from the fact that $\phi$ obeys the Bargmann-Wigner equations. The Proca equation for $A^{\mu}$ follows by inserting (3.3) into (3.2). Alternatively we may use (3.2) to eliminate $A^{\mu}$, and obtain

or equivalently

$$
p^{v} p_{\alpha} F^{\mu \alpha}-p^{\mu} p_{\beta} F^{\nu \beta}=m^{2} F^{\mu \nu},
$$

$$
\begin{aligned}
\left(p^{2}-m^{2}\right) F^{\mu v} & =0 \\
\varepsilon_{\alpha \beta \mu \nu} p^{\beta} F^{\mu v} & =0 \\
F^{\mu v}+F^{v \mu} & =0 .
\end{aligned}
$$


To derive the Takahashi-Palmer equations, we use a different expansion of the symmetric second rank spinor

$$
\phi=\operatorname{im} \gamma_{\lambda} C A^{\lambda}+\frac{1}{2} \gamma^{5} \sigma_{\lambda \nu} C G^{\lambda \nu},
$$

in which $A^{\lambda}$ is a four vector and $G^{\lambda v}$ is an anti-symmetric tensor. The validity of this expansion rests on the fact that $\gamma_{\lambda} C$ and $\gamma^{5} \sigma_{\mu \nu} C$ are as good a set of 10 independent symmetric Dirac matrices as $\gamma_{\lambda} C$ and $\sigma_{\mu \nu} C$. Indeed the relation

$$
\gamma^{5} \sigma_{\mu \nu}=\frac{1}{2} \varepsilon_{\mu \nu \sigma \varrho} \sigma^{\sigma \varrho}
$$

holds. The fact that $\phi$ obeys the Bargmann-Wigner equations now gives

$$
\begin{gathered}
\varepsilon_{\alpha \beta \sigma \varrho} p^{\beta} G^{\sigma \varrho}=m^{2} A_{\alpha} \\
G^{\alpha \beta}=-\frac{1}{2} \varepsilon^{\alpha \beta \varrho} p^{\sigma} A^{\varrho},
\end{gathered}
$$

which imply

$$
\left(p^{2}-m^{2}\right) G^{\alpha \beta}+p^{\alpha} p_{\sigma} G^{\beta \sigma}-p_{\sigma} p^{\beta} G^{\alpha \sigma}=0,
$$

or, equivalently

$$
\begin{aligned}
\left(p^{2}-m^{2}\right) G^{\alpha \beta} & =0 \\
p_{\alpha} G^{\alpha \beta} & =0 \\
G^{\alpha \beta}+G^{\beta \alpha} & =0 .
\end{aligned}
$$

The fact that (3.5) and (3.8) stem from the same Bargmann-Wigner equations makes manifest their equivalence, which has also been proved by Takahashi and Palmer, but in a different way. Of course it follows from (3.6) that $G$ is just the dual of $F$. Explicitly we have

$$
F_{\sigma \varrho}=\frac{1}{2} \varepsilon_{\sigma \varrho \mu \nu} G^{\mu \nu} \text {. }
$$

We now apply the method of Aurilia and Umezawa to derive single equations which are equivalent to the sets (3.5) and (3.8).

Let the six projectors in $(2.2),(2.3)$ be denoted by $(i=1, \ldots, 6)$, then for a given spin, $\Lambda$ defined by (1.2) can be written as

$$
\Lambda\left(p^{2}\right)=-\left(p^{2}-m^{2}\right) P_{i}-m^{2} \sum_{k \neq i} a_{k} P_{k}
$$

where $P_{i}$ is a projector for the given spin and where the $G_{k}$ are non-zero numbers. Also the Klein-Gordan divisor is given by

$$
d(p)=P_{i}+\frac{p^{2}-m^{2}}{m^{2}} \sum_{k \neq i} \frac{1}{a_{k}} P_{k} .
$$

Now, (1.1) is satisfied identically and the Klein-Gordan equation and the subsidiary conditions follow from (1.2) in virtue of the properties of the $P_{i}$. 
For the case of $P_{i}=A(1)$, the specification of the corresponding wave equation is completed by chosing the $a_{i}$ in such a way that singular terms of (3.9) cancel out. Taking the coefficient of $A^{\prime}(1)=-1$ means that singular terms of the type

$$
\frac{1}{2 p^{2}}\left(g_{\varrho}^{v} p^{\mu} p_{\sigma}+g_{\sigma}^{\mu} p^{v} p_{\varrho}-g_{\varrho}^{\mu} p^{v} p_{\sigma}-g_{\sigma}^{v} p^{\mu} p_{\varrho}\right),
$$

cancel, and taking the coefficients of $D(2), D(1), D(0)$ and $T(0)$ equal (to $b$, say; $b \neq 0$ ) suffices to cancel all other singular terms. This yields

$$
\begin{aligned}
\Lambda(p)_{\sigma \varrho}^{\mu v}= & -\frac{1}{2}\left(p^{2}-m^{2}\right)\left(g_{\sigma}^{\mu} g_{\varrho}^{v}-g_{\varrho}^{\mu} g_{\sigma}^{v}\right) \\
& +\frac{1}{2}\left(g_{\varrho}^{v} p^{\mu} p_{\sigma}+g_{\sigma}^{\mu} p^{v} p_{\varrho}-g_{\varrho}^{\mu} p^{v} p_{\sigma}-g_{\sigma}^{v} p^{\mu} p_{\varrho}\right) \\
& -\frac{1}{2} b m^{2}\left(g_{\sigma}^{\mu} g_{\varrho}^{v}+g_{\varrho}^{\mu} g_{\sigma}^{v}\right),
\end{aligned}
$$

and we also obtain

$$
\begin{aligned}
d(p)_{\sigma \varrho}^{\mu \nu}= & \frac{1}{2}\left(g_{\sigma}^{\mu} g_{\varrho}^{v}-g_{\varrho}^{\mu} g_{\sigma}^{v}\right) \\
& -\frac{1}{2 m^{2}}\left(g_{\varrho}^{v} p^{\mu} p_{\sigma}+g_{\sigma}^{\mu} p^{v} p_{\varrho}-g_{\varrho}^{\mu} p^{v} p_{\sigma}-g_{\sigma}^{v} p^{\mu} p_{\varrho}\right) \\
& +\frac{\left(p^{2}-m^{2}\right)}{2 b m^{2}}\left(g_{\sigma}^{\mu} g_{\varrho}^{v}+g_{\varrho}^{\mu} g_{\sigma}^{v}\right) .
\end{aligned}
$$

Now a Langrangian density

$$
\mathscr{L}=\psi_{\mu \nu}^{+} \Lambda_{\sigma \varrho}^{\mu v} \psi^{\sigma \varrho}
$$

yields the Euler-Lagrangian equation

$$
\Lambda_{\sigma \varrho}^{\mu v} \psi^{\sigma \varrho}=0
$$

in straightforward fashion for no anti-symmetry condition need be imposed on $\psi$ beforehand. Indeed with $\Lambda(p)$ given by (3.11), Eq. (3.13) not only implies $\psi^{\mu v}+\psi^{v \mu}=0$ but also implies $p_{\mu} \psi^{\mu v}=0$, and hence reduces to $\left(p^{2}-m^{2}\right) \psi^{\mu \nu}=0$. Thus (3.13), with (3.11), is equivalent to the Takahashi-Palmer equation (3.8). In fact, these authors derived an equation which is the $b=1$ special case of (3.13) with (3.11), and showed that (3.8) followed.

A similar analysis can be performed in the case of $P_{i}=A^{\prime}(1)$. It yields a single equation of the type of (3.13) with $\Lambda^{\prime}(p)$ given by

$$
\begin{aligned}
\Lambda^{\prime}(p)_{\sigma \varrho}^{\mu \nu}= & \frac{1}{2} m^{2}\left(g_{\sigma}^{\mu} g_{\varrho}^{v}-g_{\varrho}^{\mu} g_{\sigma}^{v}\right) \\
& -\frac{1}{2}\left(g_{\varrho}^{v} p^{\mu} p_{\sigma}+g_{\sigma}^{\mu} p^{v} p_{\varrho}-g_{\varrho}^{\mu} p^{v} p_{\sigma}-g_{\sigma}^{v} p^{\mu} p_{\varrho}\right) \\
& -\frac{1}{2} b^{\prime} m^{2}\left(g_{\sigma}^{\mu} g_{\varrho}^{v}+g_{\varrho}^{\mu} g_{\sigma}^{v}\right) .
\end{aligned}
$$


This equation implies the subsidiary conditions $\psi^{\mu v}+\psi^{v \mu}=0$ and $\varepsilon_{\alpha \beta \mu \nu} p^{\beta} \psi^{\mu \nu}=0$ and hence reduces to the Klein-Gordan equation, so that equivalence to the Proca description (3.5) is seen. The Klein-Gordan divisor is then

$$
\begin{aligned}
d^{\prime}(p)_{\sigma \varrho}^{\mu v}= & \frac{\left(p^{2}-m^{2}\right)}{2 m^{2}}\left(g_{\sigma}^{\mu} g_{\varrho}^{v}-g_{\varrho}^{\mu} g_{\sigma}^{v}\right) \\
& +\frac{1}{2 m^{2}}\left(g_{\varrho}^{v} p^{\mu} p_{\sigma}+g_{\sigma}^{\mu} p^{v} p_{\varrho}-g_{\varrho}^{\mu} p^{v} p_{\sigma}-g_{\sigma}^{v} p^{\mu} p_{\varrho}\right) \\
& +\frac{\left(p^{2}-m^{2}\right)}{2 b^{\prime} m^{2}}\left(g_{\sigma}^{\mu} g_{\varrho}^{v}+g_{\varrho}^{\mu} g_{\sigma}^{v}\right)
\end{aligned}
$$

so that in this case, as in the previous one, the Feynman propagator is known explicitly in the form

$$
\frac{d^{\prime}(p)}{p^{2}-m^{2}}
$$

In view of the dual relationship between the two formulations of spin-1, it was not necessary to apply the Aurilia-Umezawa procedure twice. Indeed we have for $A(1), A^{\prime}(1), \Lambda, \Lambda^{\prime}$, given by Eqs. (2.14), (2.15), (3.11), (3.14)

$$
\begin{aligned}
& \frac{1}{4} \varepsilon_{\mu \nu \alpha \beta} A^{\prime}(1)_{\gamma \delta}^{\alpha \beta} \varepsilon^{\gamma \delta \sigma \varrho}=A(1)_{\mu \nu}^{\sigma \varrho}, \\
& \frac{1}{4} \varepsilon_{\mu \nu \alpha \gamma} A(1)_{\gamma \delta}^{\alpha \beta} \varepsilon^{\gamma \delta \sigma \varrho}=A^{\prime}(1)_{\mu \nu}^{\sigma \varrho},
\end{aligned}
$$

and

$$
\frac{1}{4} \varepsilon_{\mu \nu \alpha \beta} \tilde{\Lambda}_{\gamma \delta}^{\alpha \beta} \varepsilon^{\gamma \delta \sigma \varrho}=\tilde{\Lambda}_{\mu \nu}^{\prime \sigma \varrho},
$$

where $\tilde{\Lambda}, \tilde{\Lambda}^{\prime}$ are defined by the equations

$$
\begin{aligned}
& \Lambda=\tilde{\Lambda}+b S, \\
& \Lambda^{\prime}=\tilde{\Lambda}^{\prime}+b^{\prime} S .
\end{aligned}
$$

It is not surprising that the dual relationship involves only the antisymmetric part of the kernels and not the full kernels since these occur in Lagrangians in which the tensor $\psi^{\mu \nu}$ is not a priori anti-symmetric.

\section{Spin-2 Wave Equation}

It was shown by Bhargava and Watanabe [14] that it is possible to construct an Euler-Lagrange equation for a massive spin-2 field in which the symmetry and the tracelessness of the tensor field employed were not assumed at the outset (cf. also Nath [16]), but emerge finally from this equation.

16 Commun. math. Phys., Vol. 24 
The set of equations which define a spin-2 field are

$$
\begin{aligned}
\left(p^{2}-m^{2}\right) \psi^{\mu v} & =0 \\
p_{\mu} \psi^{\mu v} & =0 \\
g_{\mu \nu} \psi^{\mu v} & =0 \\
\psi^{\mu \nu}-\psi^{v \mu} & =0
\end{aligned}
$$

giving five independent components in the rest frame. In this section we use the method of Aurilia and Umezawa [2] to derive a wave equation for $\psi^{\mu \nu}$ which implies the three subsidiary conditions (4.1), and upon using these reduces to the Klein-Gordan equation. It will be seen that this method is systematic and does not involve the assumption that the Umezawa-Visconti result [8] (that the highest order of $p$ in $d(p)$ is $2 s$, where $s$ is the spin) is valid. The derivation of a spin-2 equation by Bhargava and Watanabe makes this assumption, which turns out to be valid, although it has only been proved in situations where $\Lambda(p)$ is linear in $p$, and whose range of validity, clearly wider than that for which its proof exists, but equally clearly limited, is as yet undetermined.

Using the projectors found in section two, let

$$
\Lambda(p)=-\left(p^{2}-m^{2}\right) D(2)-a_{1} m^{2} A-t m^{2} T(0)-x_{0} m^{2} D(0)-x_{1} m^{2} D(1)
$$

where all the parameters are non-zero and, for simplicity, are chosen to be real. In this case, however, they cannot be chosen in such a way that all the singular terms cancel, although the terms proportional to

$$
\frac{1}{p^{2}}\left(g_{\varrho}^{v} p^{\mu} p_{\sigma}+g_{\sigma}^{\mu} p^{v} p_{\varrho}+g_{\varrho}^{\mu} p^{v} p_{\sigma}+g_{\sigma}^{v} p^{\mu} p_{\varrho}\right)
$$

are removed by putting $x_{1}=-1$. The remaining singularities

$$
\frac{p^{\mu} p^{v} p_{\sigma} p_{\varrho}}{p^{4}}, \quad \frac{p^{\mu} p^{v} p_{\sigma} p_{\varrho}}{p^{2}}, \quad \frac{p^{\mu} p^{v} g_{\sigma \varrho}}{p^{2}}, \quad \frac{g^{\mu v} p_{\sigma} p_{\varrho}}{p^{2}}
$$

may be removed by multiplying $\Lambda$ on the left by invertible operators of the form

$$
1+c P_{k} M P_{l} ; \quad P_{k}, P_{l} \neq D(2)
$$

where $M$ is some matrix and $c$ is a suitable coefficient, chosen so that the singularities generated by $P_{k} M P_{l}$ cancel those in $\Lambda$. Consider

$$
\begin{gathered}
X_{\sigma \varrho}^{\mu \nu} \equiv D(0)_{\alpha \beta}^{\mu \nu} \frac{p^{\alpha} p^{\beta}}{p^{2}} g_{\xi \eta} T(0)_{\sigma \varrho}^{\xi \eta}=-\frac{1}{4} g^{\mu v} g_{\sigma \varrho}+\frac{p^{\mu} p^{v}}{p^{2}} g_{\sigma \varrho}, \\
Y_{\sigma \varrho}^{\mu \nu} \equiv T(0)_{\alpha \beta}^{\mu \nu} g^{\alpha \beta} \frac{p_{\xi} p_{\eta}}{p^{2}} D(0)_{\sigma \varrho}^{\xi \eta}=-\frac{1}{4} g^{\mu v} g_{\sigma \varrho}+g^{\mu \nu} \frac{p_{\sigma} p_{\varrho}}{p^{2}},
\end{gathered}
$$




$$
\begin{aligned}
& X_{\alpha \beta}^{\mu v} Y_{\sigma \varrho}^{\alpha \beta}=\frac{1}{4} g^{\mu v} g_{\sigma \varrho}-g^{\mu v} \frac{p_{\sigma} p_{\varrho}}{p^{2}}-\frac{p^{\mu} p^{v} g_{\sigma \varrho}}{p^{2}}+4 \frac{p^{\mu} p^{v} p_{\sigma} p_{\varrho}}{p^{4}}, \\
& Y_{\alpha \beta}^{\mu v} X_{\sigma \varrho}^{\alpha \beta}=3 / 4 g^{\mu v} g_{\sigma \varrho} .
\end{aligned}
$$

All the singularities can therefore be generated by multiplying $\Lambda$ on the left by $\eta_{2} \eta_{1}$ where

$$
\begin{aligned}
& \eta_{2}=1+\left(c \frac{p^{2}}{m^{2}}+d\right) X, \\
& \eta_{1}=1+\left(a \frac{p^{2}}{m^{2}}+b\right) Y .
\end{aligned}
$$

Not all of the four parameters $a, b, c, d$ are necessary for the purpose of eliminating singular terms, and it is possible to have either $a=0$ or $c=0$; but the latter leads to a non-hermitian Lagrangian density. Consequently we set $a=0$.

Now in order to have the hermiticity of the Lagrangian compatible with the vanishing of the singular terms, it is necessary to multiply by another operator

It follows that

$$
\eta_{3}=1+q Y
$$

$$
\begin{aligned}
\tilde{\Lambda}_{\sigma \varrho}^{\mu v}= & \left(\eta_{3} \eta_{2} \eta_{1}\right)_{\alpha \beta}^{\mu v} \Lambda_{\sigma \varrho}^{\alpha \beta} \\
= & -\frac{1}{2}\left(p^{2}-m^{2}\right)\left(g_{\sigma}^{\mu} g_{\varrho}^{v}+g_{\varrho}^{\mu} g_{\sigma}^{v}\right)-a_{1} m^{2} A_{\sigma \varrho}^{\mu \nu} \\
& +\frac{1}{2}\left(g_{\varrho}^{v} p^{\mu} p_{\sigma}+g_{\sigma}^{\mu} p^{v} p_{\varrho}+g_{\varrho}^{\mu} p^{v} p_{\sigma}+g_{\sigma}^{v} p^{\mu} p_{\varrho}\right) \\
& +p^{2} g^{\mu v} g_{\sigma \varrho}\left[\frac{1}{2}+\frac{1}{4} c t-\frac{1}{4} b c x_{0}+\frac{3}{4} q c\left(-t+b x_{0}\right)\right] \\
& +m^{2} g^{\mu v} g_{\sigma \varrho}\left[-\frac{1}{4} t-\frac{1}{3}-\frac{1}{12} x_{0}+\frac{1}{4} b x_{0}+\frac{1}{4} d t-\frac{1}{4} b d x_{0}\right. \\
& \left.\quad+q\left(\frac{1}{4} x_{0}-\frac{3}{4} d t+\frac{3}{4} b d x_{0}\right)\right] \\
& +g^{\mu v} p_{\sigma} p_{\varrho}\left[-\frac{1}{3}+b c x_{0}-3 q b c x_{0}\right] \\
& +p^{\mu} p^{v} g_{\sigma \varrho}\left[-\frac{1}{3}-c t+b c x_{0}\right] \\
& +m^{2} \frac{p^{\mu} p^{v} g_{\sigma \varrho}}{p^{2}}\left[\frac{1}{3}+\frac{1}{3} x_{0}+b d x_{0}-d t\right] \\
& +m^{2} \frac{g^{\mu v} p_{\sigma} p_{\varrho}}{p^{2}}\left[\frac{1}{3}+\frac{1}{3} x_{0}-b x_{0}+b d x_{0}-q x_{0}(1+3 b d)\right] \\
& +m^{2} \frac{p^{\mu} p^{v} p_{\sigma} p_{\varrho}}{p^{4}}\left[-\frac{4}{3}-\frac{4}{3} x_{0}-4 b d x_{0}\right] \\
& +\frac{p^{\mu} p^{v} p_{\sigma} p_{\varrho}}{p^{2}}\left[-\frac{2}{3}-4 b c x_{0}\right] .
\end{aligned}
$$


The singularities are removed by requiring that the coefficients of the last four terms vanish, and the hermiticity of the Lagrangian is assured by requiring that the coefficients of $g^{\mu v} p_{\sigma} p_{\varrho}$ and $p^{\mu} p^{v} g_{\sigma \varrho}$ are equal. Thus (4.5) gives

$$
b c x_{0}=-\frac{1}{6}
$$

so that hermiticity demands that

Put

$$
c t=-\frac{1}{2} q .
$$

$$
\begin{aligned}
\alpha & =-\frac{1}{3}-c t+b c x_{0} \\
& =-\frac{1}{2}+\frac{1}{2} q
\end{aligned}
$$

and

$$
\begin{aligned}
\beta & =\frac{1}{3}+\frac{1}{4} c t-\frac{1}{4} b c x_{0}+q\left(-\frac{3}{4} c t+\frac{3}{4} b c x_{0}\right) \\
& =\frac{3}{8} q^{2}-\frac{1}{4} q+\frac{3}{8} .
\end{aligned}
$$

It follows that

$$
\beta=\frac{1}{2}\left(3 \alpha^{2}+2 \alpha+1\right) .
$$

Now (4.2) and (4.4) give $d=0$ and $x_{0}=-1$, and hence (4.3) demands that $b+q=0$. Putting

$$
\begin{aligned}
\gamma= & \frac{1}{4} t+\frac{1}{3}+\frac{1}{12} x_{0}-\frac{1}{4} b x_{0}-\frac{1}{4} d t+\frac{1}{4} b d x_{0} \\
& -q\left(\frac{1}{4} x_{0}-\frac{3}{4} d t+\frac{3}{4} b d x_{0}\right)
\end{aligned}
$$

then

$$
\gamma=\frac{1}{4}\left(3 q^{2}+1\right)=\alpha+2 \beta .
$$

The resulting $\tilde{\Lambda}$, depending on two parameters $\left(\alpha, a_{1}\right)$ is then

$$
\begin{aligned}
\tilde{\Lambda}_{\sigma \varrho}^{\mu v}= & -\frac{1}{2}\left(p^{2}-m^{2}\right)\left(g_{\sigma}^{\mu} g_{\varrho}^{v}+g_{\varrho}^{\mu} g_{\sigma}^{v}\right)-a_{1} m^{2} A_{\sigma \varrho}^{\mu v} \\
& +\frac{1}{2}\left(g_{\varrho}^{v} p^{\mu} p_{\sigma}+g_{\sigma}^{\mu} p^{v} p_{\varrho}+g_{\varrho}^{\mu} p^{v} p_{\sigma}+g_{\sigma}^{v} p^{\mu} p_{\varrho}\right) \\
& +\alpha\left(g^{\mu v} p_{\sigma} p_{\varrho}+p^{\mu} p^{v} g_{\sigma \varrho}\right)+\beta p^{2} g^{\mu v} g_{\sigma \varrho} \\
& -\gamma m^{2} g^{\mu v} g_{\sigma \varrho}
\end{aligned}
$$

where $\beta$ and $\gamma$ are defined by (4.6) and (4.7). Since $q \neq 0$ then $\alpha \neq-\frac{1}{2}$; and, of course, $a_{1} \neq 0$.

The Klein-Gordan divisor, defined by

where

$$
\tilde{d}=d \eta_{1}^{-1} \eta_{2}^{-1} \eta_{3}^{-1}
$$

$$
d=D(2)+\frac{\left(p^{2}-m^{2}\right)}{m^{2}}\left[a_{1}^{-1} A+t^{-1} T(0)+x_{0}^{-1} D(0)+x_{1}^{-1} D(1)\right]
$$


turns out to be

$$
\begin{aligned}
\tilde{d}_{\sigma \varrho}^{\mu \nu}= & \frac{1}{2}\left(g_{\sigma}^{\mu} g_{\varrho}^{v}+g_{\varrho}^{\mu} g_{\sigma}^{v}\right)-\frac{1}{3} g^{\mu v} g_{\sigma \varrho}+\frac{1}{3 m^{2}}\left(p^{\mu} p^{v} g_{\sigma \varrho}+g^{\mu v} p_{\sigma} p_{\varrho}\right) \\
& +\frac{2}{3} \frac{p^{\mu} p^{v} p_{\sigma} p_{\varrho}}{m^{4}}-\frac{1}{2 m^{2}}\left(g_{\varrho}^{v} p^{\mu} p_{\sigma}+g_{\sigma}^{\mu} p^{v} p_{\varrho}+g_{\varrho}^{\mu} p^{v} p_{\sigma}+g_{\sigma}^{v} p^{\mu} p_{\varrho}\right) \\
& +\frac{\left(p^{2}-m^{2}\right)}{m^{2}}\left[-\frac{1}{3} \frac{\alpha(\alpha+1)}{(2 \alpha+1)^{2}} g^{\mu v} g_{\sigma \varrho}+\frac{(\alpha+1)^{2}}{6(2 \alpha+1)^{2}} \frac{p^{2}}{m^{2}} g^{\mu v} g_{\sigma \varrho}\right. \\
& \left.-\frac{1}{3} \frac{(\alpha+1)}{(2 \alpha+1)}\left(p^{\mu} p^{v} g_{\sigma \varrho}+g^{\mu v} p_{\sigma} p_{\varrho}\right)+a_{1}^{-1} A_{\sigma \varrho}^{\mu v}\right] .
\end{aligned}
$$

$\tilde{\Lambda}$ and $\tilde{d}$ are the same as those derived in Ref. [14], and the Lagrangian density and propagator are given by

$$
\begin{aligned}
\mathscr{L}(x) & =\psi_{\mu \nu}^{+}(x) \tilde{\Lambda}_{\sigma \varrho}^{\mu \nu} \psi^{\sigma \varrho}(x) \\
\mathscr{P}_{\sigma \varrho}^{\mu \nu}(p) & =\frac{\tilde{d}_{\sigma \varrho}^{\mu \nu}(p)}{p^{2}-m^{2}} .
\end{aligned}
$$

It is to be noted that $\Lambda$ in section three corresponding to particles described eventually by an anti symmetric tensor involves the projector onto symmetric components in the simple form

$$
-b m^{2} S, \quad b \neq 0 .
$$

Also $\tilde{\Lambda}$ in section four which corresponds to particles eventually described by a symmetric tensor involves the projector onto anti symmetric components in a correspondingly simple fashion

$$
-a_{1} m^{2} A, \quad a_{1} \neq 0 .
$$

Further, the procedure involving the operators $\eta$, which is the essential new feature in the Aurilia and Umezawa approach, is non trivial only for spin zero components of the symmetric part of $\psi^{\mu \nu}$. Remarks analogous to these will serve to minize the effort involved in applying the Aurilia-Umezawa approach to more complicated situations, some of which are currently under investigation.

One of the authors (W.T.) is indebted to the Carnegie Trust for the Universities of Scotland for financial support.

\section{References}

1. Aurilia, A., Umezawa, H.: Nuovo Cimento A 51, 14 (1967).

2. - - Phys. Rev. 182, 1682 (1969).

3. Dirac, P. A. M.: Proc. Roy. Soc. (Lond.) A 155, 447 (1936).

4. Fierz, M.: Helv. Phys. Acta 12, 3 (1939). 
5. - Pauli, W.: Proc. Roy. Soc. (Lond.) A 173, 211 (1939).

6. Rarita, W., Schwinger, J.: Phys. Rev. 60, 61 (1940).

7. Takahashi, Y., Umezawa,H.: Prog. Theoret. Phys. (Kyoto) 9, 1 (1953); Nucl. Phys. 51, 193 (1964).

8. Umezawa, H., Visconti, A.: Nucl. Phys. 1, 348 (1956).

9. Lurie,D., Takahashi, Y., Umezawa,H.: J. Math. Phys. 7, 1478 (1966).

10. Fronsdal, C.: Nuovo Cimento Suppl. 9, 416 (1958).

11. Chang, S. J.: Phys. Rev. 161, 1308 (1967).

12. Takahashi, Y., Palmer, R.: Phys. Rev. D 1, 2974 (1970).

13. Bargmann, W., Wigner, E.P.: Proc. Nat. Acad. Sci. V.S. 34, 211 (1948).

14. Bhargava, S.C., Watanabe, H.: Nucl. Phys. 87, 273 (1966).

15. Rivers, R.J.: Nuovo Cimento 34, 386 (1966).

16. Lurié, D.: Particles and Fields. New York: Wiley 1968.

17. Nath, L. M.: Nucl. Phys. 68, 660 (1965).

18. Kamefuchi, S., Takahashi, Y.: Nuovo Cimento 44, 1 (1968).

\author{
W. Tait \\ Institut Henri Poincaré \\ 11 Rue Pierre Curie \\ F-75 Paris Ve $\mathrm{e}^{\mathrm{e}}$ France
}

